\title{
Ketogenic diet increases concentrations of kynurenic acid in discrete brain structures of young and adult rats
}

\author{
Tomasz Żarnowski • Tomasz Choragiewicz • Maria Tulidowicz-Bielak • \\ Sebastian Thaler $\cdot$ Robert Rejdak $\cdot$ Iwona Żarnowska $\cdot$ \\ Waldemar Andrzej Turski • Maciej Gasior
}

Received: 23 October 2011/Accepted: 1 December 2011/Published online: 27 December 2011

(C) The Author(s) 2011. This article is published with open access at Springerlink.com

\begin{abstract}
Targeting mechanisms that result in increased concentrations of kynurenic acid (KYNA) in the brain has been considered as a therapeutic approach for the treatment of epilepsy and certain neurodegenerative disorders. Recently, KYNA has been implicated in the effects produced by the high-fat and low-protein/carbohydrate ketogenic diet (KD) in a report demonstrating an increased production of KYNA in vitro by one of the ketone bodies, $\beta$-hydroxybutyrate, elevated by the KD. To further explore this association, brain concentrations of KYNA were compared in young ( 3 weeks old) and adult (8-10 weeks old) rats that were chronically exposed to the KD and regular diet. Exposure to the $\mathrm{KD}$ resulted in the anticipated elevations of $\beta$ -
\end{abstract}

T. Żarnowski · T. Chorągiewicz - M. Tulidowicz-Bielak ·

R. Rejdak

Department of Ophthalmology, Medical University,

Lublin, Poland

S. Thaler

Centre for Ophthalmology, University of Tübingen,

Tübingen, Germany

I. Żarnowska

SANITAS, Private Medical Centre, Lublin, Poland

W. A. Turski

Department of Toxicology, Institute of Agricultural Medicine,

Lublin, Poland

W. A. Turski

Department of Experimental and Clinical Pharmacology,

Medical University, Lublin, Poland

Present Address:

M. Gasior $(\square)$

Discovery Medicine, Neuroscience,

Bristol-Myers Squibb Company, Princeton, NJ 08543, USA

e-mail: maciej.gasior@bms.com; NextPharma@gmail.com hydroxybutyrate with accompanying decreases in glucose concentrations. In comparison to rats fed the regular diet, KYNA concentrations were significantly $(p<0.05)$ increased in the hippocampus (256 and 363\% increase in young and adult rats, respectively) and in the striatum (381 and $191 \%$ increase in young and adult rats, respectively) in KD-fed rats. KD-induced increases in KYNA concentrations in young versus adult rats in the hippocampus and striatum were comparable $(p>0.05)$. Exposure to the KD had no effect on KYNA concentrations in the cortex of young and adult rats $(p>0.05)$. In summary, chronic exposure to the KD resulted in several-fold increases in KYNA concentrations in discrete brain structures in the rats. Thus, the relevant clinical question for further exploration is whether $\mathrm{KD}$ induced increases in KYNA concentrations can translate into clinically significant improvements in neuropsychiatric diseases associated with KYNA hypofunction.

Keywords Ketogenic diet - Kynurenic acid - Brain . Epilepsy $\cdot$ Neurodegenerative disorders · Cortex · Hippocampus · Striatum

$\begin{array}{ll}\text { Abbreviations } \\ \text { AMPA } & \begin{array}{l}\text { 2-Amino-3-(5-methyl-3-oxo-1,2-oxazol-4- } \\ \text { yl)propanoic acid }\end{array} \\ \text { KD } & \text { Ketogenic diet } \\ \text { KYNA } & \text { Kynurenic acid } \\ \text { NMDA } & N \text {-Methyl-D-aspartate }\end{array}$

\section{Introduction}

Kynurenic acid (KYNA) is a metabolite of tryptophan and is formed by an irreversible transamination of its metabolic intermediate, kynurenine, with the aid of kynurenine 
aminotransferases (KATs) that are present in the periphery as well as in the brain (Zadori et al. 2009). Accumulated evidence points to the specific pharmacological actions of KYNA, inducible enzymatic control of its production, and consequent roles in several neuropsychiatric disorders (Erhardt et al. 2009; Vamos et al. 2009; Kincses et al. 2010).

For example, KYNA has been demonstrated to block the $\mathrm{N}$-methyl-D-aspartate (NMDA), 2-amino-3-(5-methyl-3oxo-1,2-oxazol-4-yl)propanoic acid (AMPA), kainate, and $\alpha 7$-nicotinic acetylcholine receptors at $\mathrm{nM}$ to $\mu \mathrm{M}$ concentrations (Zadori et al. 2009). Furthermore, changes in KYNA concentrations have been implicated as causative or compensatory in epilepsy and several neurodegenerative disorders such as Parkinson's, Alzheimer's, and Huntington's diseases (Erhardt et al. 2009; Zadori et al. 2009; Vamos et al. 2009; Kincses et al. 2010). Thus, targeting mechanisms that result in increased concentrations of endogenous KYNA have been considered as a therapeutic approach for the treatment of epilepsy and certain neurodegenerative disorders.

These disorders might also benefit from therapies such as the high-fat and low-protein/carbohydrate ketogenic diet (KD) that interfere with glucose and ketone metabolism (Gasior et al. 2006; Freeman and Kossoff 2010). Recently, two studies explicitly provided the in vitro evidence implicating KYNA in the effects produced by the KD. First, NMDA-induced reduction of KYNA production was reversed by two ketone bodies, acetoacetate and $\beta$ hydroxybutyrate, elevated during the exposure to the KD in bovine retinal slices (Thaler et al. 2010). Second, $\beta$ hydroxybutyrate increased the synthesis of KYNA in brain cortical slices and primary glial cultures (Chmiel-Perzynska et al. 2011). However, one limitation of these in vitro studies is that they do not model complex consequences of chronic exposure to the $\mathrm{KD}$, such as ketosis caused by elevated concentrations of all three ketone bodies (i.e., acetone, acetoacetate, and $\beta$-hydroxybutyrate), accompanying carbohydrate deprivation with resultant decreases in glucose concentrations, and various biological effects taking place at the cellular and organ levels (Bough et al. 2006; Hartman et al. 2007; Maalouf et al. 2009).

Thus, the aim of the present study was to explore the association of KYNA and the KD in vivo by employing a rat model of chronic exposure to the KD that has been broadly used in KD-related research, owing to its translational validity (Stafstrom 1999; Bough et al. 2002; Hartman et al. 2007). Specifically, concentrations of KYNA were measured in discrete brain regions of rats chronically exposed to the KD and regular diet for comparison. KYNA concentrations were further compared in young and adult rats since responses to the KD have been reported to be age dependent (Bough et al. 1999; Prins and Hovda 2009).
Based on the available in vitro reports, we predicted that exposure to the KD would increase brain concentrations of KYNA in vivo.

\section{Material and methods}

Animals and exposure to the KD

Female Brown Norway rats (Charles River, Wilmington, MA) weighing 50-70 g (approximately, 3 weeks old and considered as young) or 125-150 g (approximately, 8-10 weeks old and considered as adult) were used in the present study. They were housed under a 12-h light-dark cycle (with standard controlled humidity and temperature values).

The rats were fed ad libitum with the KD (F3666, AIN76A-modified high-fat paste, BioServ, Frenchtown, NJ, USA) for 21 consecutive days. The KD was composed of approximately $78.8 \%$ fat, $8.4 \%$ protein, $5 \%$ cellulose, less than $5 \%$ water and less than $1 \%$ carbohydrate, and was supplemented with necessary minerals and amino acids. Control rats received standard rodent diet (3430 Kliba Nafag, Switzerland). On the 21st day of exposure to the $\mathrm{KD}$ (or the regular diet in the control rats), rats' brains were collected and immediately frozen in liquid nitrogen for further KYNA concentration determination.

\section{Brain concentrations of KYNA}

KYNA concentrations were measured in the cortex, striatum, and hippocampus by means of high-performance liquid chromatography (HPLC) with fluorescence detection as described elsewhere (Turski et al. 1988; Shibata 1988). In brief, specimens were sonicated in $2 \mathrm{vol}(\mathrm{w} / \mathrm{v})$ of distilled water, immersed in boiling water bath for $10 \mathrm{~min}$, and centrifuged for $10 \mathrm{~min}$ at 20,000 rpm. The resulting supernatant was then diluted (1:1) with $0.2 \mathrm{~N} \mathrm{HCl}$ and applied to Dowex $50-\mathrm{W}$ hydrogen form prewashed with $0.1 \mathrm{~N} \mathrm{HCl}$. Columns were subsequently washed with $1 \mathrm{ml} 0.1 \mathrm{~N} \mathrm{HCl}$ and $1 \mathrm{ml}$ water. KYNA was eluted with $2 \mathrm{ml}$ of water. The eluate was subjected to HPLC and detected fluorometrically as described elsewhere (Shibata 1988). KYNA concentrations (in $\mathrm{pmol} / \mathrm{g}$ wet tissue weight) were expressed as group means ( \pm SEM; $n=4-6 /$ each structure).

Plasma concentrations of glucose and $\beta$-hydroxybutyrate

Plasma concentrations of glucose $(\mathrm{mg} / \mathrm{dL})$ and $\beta$ hydroxybutyrate $(\mathrm{mM})$ were measured in selected animals to confirm that ketosis with accompanying hypoglycemia developed in rats maintained on the $\mathrm{KD}$; control rats that 
were fed a regular diet were included for comparison (Hartman et al. 2008). Blood samples were collected by making a small incision on the tail's skin at the time corresponding to the tissue collection for the measurements of KYNA concentrations. Immediately following sampling, glucose and $\beta$-hydroxybutyrate concentrations were measured using a test strip system and reader (Precision Xtra Advanced Diabetes Management System with Precision Xtra blood ketone test strips and blood glucose test strips; Abbott Diabetes Care Inc., Alameda, CA) as described elsewhere (Hartman et al. 2008). Plasma concentrations of glucose and $\beta$-hydroxybutyrate were expressed as group means ( \pm SEM; $n=4$ /group).

\section{Statistical analysis}

Statistical analysis was performed using unpaired Student's $t$-test (plasma concentrations of glucose and $\beta$-hydroxybutyrate) or two-way analysis of variance (ANOVA; brain concentrations of KYNA). When appropriate, post hoc analysis was performed using Tukey test. Differences were considered statistically significant at $p<0.05$.

\section{Results}

Effect of the KD on plasma glucose and

$\beta$-hydroxybutyrate concentrations in young and adult rats

In comparison to the young rats maintained on a regular diet, a 21-day exposure to the KD resulted in significant changes in plasma concentrations of glucose (decrease from $104.0 \pm 8.7$ to $49.0 \pm 13.6 \mathrm{mg} / \mathrm{dL}$, respectively; $p=$ 0.014 ) and $\beta$-hydroxybutyrate (increase from $0.68 \pm 0.15$ to $5.53 \pm 1.21 \mathrm{mM}$, respectively; $p=0.007)$. In comparison to the adult rats maintained on the regular diet, a 21-day exposure to the $\mathrm{KD}$ resulted in significant changes in plasma concentrations of glucose (decrease from $124.5 \pm 8.5$ to $59.0 \pm 10.4 \mathrm{mg} / \mathrm{dL}$, respectively; $p=0.003)$ and $\beta$ hydroxybutyrate (increase from $0.75 \pm 0.03$ to $4.40 \pm$ $0.90 \mathrm{mM}$, respectively; $p=0.007$ ). In young versus adult rats, the magnitude of the decrease in plasma glucose concentrations was comparable $(52.9 \% \pm 13.09$ and $52.6 \% \pm$ 8.3 , respectively; $p=0.985$ ). In young versus adult rats, the magnitude of the increase in plasma $\beta$-hydroxybutyrate concentrations was comparable $(818.5 \% \pm 178.5$ and $586.7 \% \pm 120.1$, respectively; $p=0.323$ ).

Effect of the KD on brain concentrations of KYNA in young and adult rats

Two-way ANOVA indicated statistically significant treatment effect $\left(F_{3,47}=17.319 ; p<0.001\right)$ after aggregated

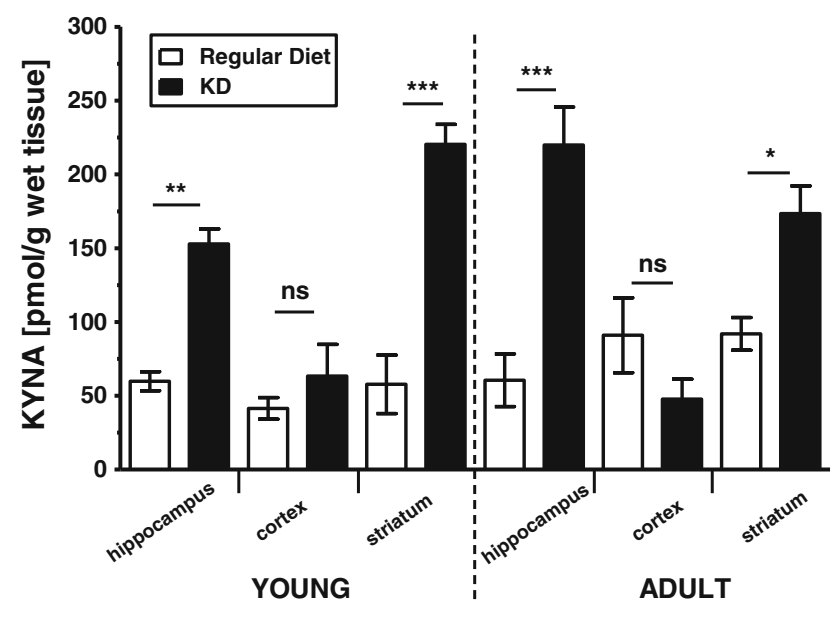

Fig. 1 Concentrations (mean $\pm \mathrm{SEM} ; n=3-6 /$ each structure) of KYNA (pmol/g wet tissue) in the hippocampus, cortex, and striatum in young and adult rats fed a regular diet (open bars) or the KD (solid bars). Asterisks represent significant difference in the KD versus standard diet-fed rats at $p<0.05(*), p<0.01(* *)$, or $p<0.001$ $(* * *)$; ns donates non-significant difference (Tukey test following significant ANOVA)

data for brain concentrations of KYNA across different structures passed normality $(p=0.090)$ and equal variance $(p=0.643)$ tests. Post hoc analysis (Tukey test) in rats maintained on the regular diet (Fig. 1) revealed that concentrations of KYNA in young and adult rats were comparable in the hippocampus $(p=1.0)$, cortex $(p=0.371)$, and striatum $(p=0.615)$. Likewise, concentrations of KYNA in young and adult rats fed with the KD for 21 days were comparable in these structures (i.e., hippocampus, $p=0.50$; cortex, $p=0.937$; and striatum, $p=0.221$ ).

Regardless of the age, a 21-day exposure to the KD resulted in significant increases in KYNA concentrations in the hippocampus (young rats: $p=0.009$; adult rats: $p<0.001$ ) and striatum (young rats: $p<0.001$; adult rats; $p=0.012)$ in KD-fed rats in comparison to the control rats fed a regular diet (Tukey test; Fig. 1). Specifically, exposure to the KD resulted in a 256 and $363 \%$ increase in concentrations of KYNA in the hippocampus of the young and adult rats, respectively. Furthermore, exposure to the KD resulted in a 381 and $191 \%$ increase in concentrations of KYNA in the striatum of young and adult rats, respectively. In contrast, concentrations of KYNA in the cortex were not affected by exposure to the KD in comparison to the age-matching young $(p=0.879)$ and adult $(p=0.416)$ rats fed a regular diet (Fig. 1).

\section{Discussion}

The results of the present study provide the first in vivo evidence that chronic exposure to the KD increases concentrations of KYNA in discrete brain regions (i.e., the 
hippocampus and striatum, but not cortex) in rats. This finding proposes a potential mechanism involved in the therapeutic effects of the KD, especially in neuropsychiatric disorders associated with KYNA hypofunction.

The methodology of chronic exposure to the KD used in the present study resulted in the development of chronic ketosis with corresponding hypoglycemia. These metabolic changes were consistent with those reported when the KD was studied for its anticonvulsant properties in animals (Appleton and De Vivo 1973; Greene et al. 2001; Hartman et al. 2008; Samala et al. 2011). Also, KYNA concentrations in brain structures of animals when fed a regular diet in the present study corresponded with previously published data (Gramsbergen et al. 1992). In contrast to previous reports, however, there was no age-dependent increase in brain KYNA concentrations in young versus adult rats. This lack of difference was likely due to a small difference in age in the groups used in the present study (approximately, 5-7-week difference) in comparison to earlier studies (approximately, 18-21 months difference) that reported age-dependent increases in KYNA concentrations (Moroni et al. 1988; Gramsbergen et al. 1992).

In the present study, concentrations of KYNA in the KDexposed rats increased 1.9- and 3.8-fold in the striatum and hippocampus in comparison to control rats, respectively; concentrations of KYNA in the cortex did not changed significantly. This differential effect suggests that the KD affects specific brain structures in a unique way rather than producing a global state of KYNA elevation. Alternatively, different brain regions may have different capacities and/or sensitivities to respond to the KD with resultant increases in KYNA concentrations. Of note, brain-specific changes in KYNA concentrations due to differential sensitivities of KATs to various modulators (e.g., metabolic substrates, $\mathrm{pH}$, pharmacological inhibitors, or convulsive stimulations) have been previously reported (Moroni et al. 2005; Maciejak et al. 2009; Han et al. 2010; Szyndler et al. 2011).

The effects of external factors on KYNA concentrations in vivo have rarely been reported. Thus, it is difficult to compare the magnitude of the KD-induced increases in KYNA in the present study to responses produced by other means. Administration of kainic acid at doses that produced convulsions resulted in $200-500 \%$ increases in KYNA concentrations in the piriform cortex, amygdala, and cerebellum in rats (Baran et al. 1995). An approximately $50 \%$ increase in KYNA production was reported after incubation of rat cortical slices and primary glial cultures with $\beta$-hydroxybutyrate (Chmiel-Perzynska et al. 2011). Under similar experimental conditions, increases in KYNA production did not exceed $100 \%$ after incubations with several approved antiepileptic drugs (Kocki et al. 2006). Likewise, a reduction of $\mathrm{Na}^{+}$concentration resulted in a $20-30 \%$ increase in KYNA production (Turski et al.
1989). Probably, the greatest increases in KYNA production in vitro (approximately, 155-170\%) were reported during the incubation of neuronal and glial preparations with ammonia (Saran et al. 2004; Wejksza et al. 2006).

Since excessive increases in KYNA concentration may have detrimental behavioral effects on, for example, cognition or ambulatory activity (Vecsei and Beal 1990; Potter et al. 2010), it is important to note that the KD is generally well tolerated by the animals and no gross behavioral abnormalities have been reported. In contrast, exposure to the KD has been reported to produce generally positive effects on spontaneous or disease-altered behavioral outcomes (e.g., Murphy et al. 2004; Ziegler et al. 2005; Mantis et al. 2009; Ruskin et al. 2011). Thus, increases in KYNA concentrations produced by the KD do not appear to reach levels that would result in behavioral side effects.

The exact mechanism whereby an exposure to the KD would result in increases in KYNA concentrations is not known. In vitro studies demonstrated an increased activity of KYNA-producing KATs in cultured glial cells incubated with $\beta$-hydroxybutyrate; this effect, however, was not confirmed in cortical homogenates (Chmiel-Perzynska et al. 2011). Note that KYNA concentrations were not changed by the KD in the present study. Furthermore, KYNA production was enhanced in vitro by the addition of substrates that increase cellular metabolism (Hodgkins and Schwarcz 1998). Of note, exposure to the KD, among others, increases cellular metabolism (Bough et al. 2006), thus providing a potential mechanistic explanation of the KD-induced increases in KYNA concentrations reported here.

In summary, chronic exposure to the $\mathrm{KD}$ in rats resulted in several-fold increases in KYNA concentrations in discrete brain structures. The magnitude of these increases was generally greater than increases produced by any other modulators (e.g., antiepileptic drugs) studied so far under physiological conditions. Given the proposed role of KYNA in the pathophysiology of several neuropsychiatric disorders, further studies in this area are justified. Also, the development of small molecule-based approaches targeting the kynurenine pathway to increase KYNA (e.g., via the inhibition of kynurenine hydroxylase or kynureninase) remains at the stage of pre-clinical testing. Thus, the KD may offer an alternative and expedited way of obtaining the clinical proof-of-concept evidence to test whether exogenously induced increases in KYNA concentrations in the brain can translate into clinically significant improvements in neuropsychiatric diseases associated with KYNA hypofunction. Testing of this hypothesis is supported mechanistically, since an increased cellular energy metabolism has been implicated in the effects produced by the KD and as a factor enhancing KYNA production. Further support comes from a long history of clinical benefit of the KD in drug-resistant epilepsy syndromes and the proposed clinical 
utility of the KD in other neuropsychiatric disorders (Gasior et al. 2006; Freeman and Kossoff 2010).

Acknowledgments This work was supported by the Foundation for Polish Science, Warsaw, Poland.

Conflict of interest We confirm that we have read the journal's position on issues involved in ethical publication and affirm that this report is consistent with those guidelines. M. Gasior is a full-time employee of Bristol-Myers Squibb Company at the time of this submission. However, this work has not been supported by and does not have any association with M. Gasior's current employer. The authors declare no conflict of interest.

Open Access This article is distributed under the terms of the Creative Commons Attribution Noncommercial License which permits any noncommercial use, distribution, and reproduction in any medium, provided the original author(s) and source are credited.

\section{References}

Appleton DB, De Vivo DC (1973) An experimental animal model for the effect of ketogenic diet on epilepsy. Proc Aust Assoc Neurol 10:75-80

Baran H, Gramer M, Honack D, Loscher W (1995) Systemic administration of kainate induces marked increases of endogenous kynurenic acid in various brain regions and plasma of rats. Eur J Pharmacol 286:167-175

Bough KJ, Valiyil R, Han FT, Eagles DA (1999) Seizure resistance is dependent upon age and calorie restriction in rats fed a ketogenic diet. Epilepsy Res 35:21-28

Bough KJ, Gudi K, Han FT, Rathod AH, Eagles DA (2002) An anticonvulsant profile of the ketogenic diet in the rat. Epilepsy Res 50:313-325

Bough KJ, Wetherington J, Hassel B, Pare JF, Gawryluk JW, Greene JG, Shaw R, Smith Y, Geiger JD, Dingledine RJ (2006) Mitochondrial biogenesis in the anticonvulsant mechanism of the ketogenic diet. Ann Neurol 60:223-235

Chmiel-Perzynska I, Kloc R, Perzynski A, Rudzki S, Urbanska EM (2011) Novel aspect of ketone action: beta-hydroxybutyrate increases brain synthesis of kynurenic acid in vitro. Neurotoxic Res 20:40-50

Erhardt S, Olsson SK, Engberg G (2009) Pharmacological manipulation of kynurenic acid: potential in the treatment of psychiatric disorders. CNS Drugs 23:91-101

Freeman JM, Kossoff EH (2010) Ketosis and the ketogenic diet, 2010: advances in treating epilepsy and other disorders. Adv Pediatr 57:315-329

Gasior M, Rogawski MA, Hartman AL (2006) Neuroprotective and disease-modifying effects of the ketogenic diet. Behav Pharmacol 17:431-439

Gramsbergen JB, Schmidt W, Turski WA, Schwarcz R (1992) Agerelated changes in kynurenic acid production in rat brain. Brain Res 588:1-5

Greene AE, Todorova MT, McGowan R, Seyfried TN (2001) Caloric restriction inhibits seizure susceptibility in epileptic EL mice by reducing blood glucose. Epilepsia 42:1371-1378

Han Q, Cai T, Tagle D, Li J (2010) Structure, expression, and function of kynurenine aminotransferases in human and rodent brains. Cell Mol Life Sci 67:353-368

Hartman AL, Gasior M, Vining EP, Rogawski MA (2007) The neuropharmacology of the ketogenic diet. Pediatr Neurol $36: 281-292$
Hartman AL, Lyle M, Rogawski MA, Gasior M (2008) Efficacy of the ketogenic diet in the 6-Hz seizure test. Epilepsia 49:334-339

Hodgkins PS, Schwarcz R (1998) Interference with cellular energy metabolism reduces kynurenic acid formation in rat brain slices: reversal by lactate and pyruvate. Eur J Neurosci 10:1986-1994

Kincses ZT, Toldi J, Vecsei L (2010) Kynurenines, neurodegeneration and Alzheimer's disease. J Cell Mol Med 14:2045-2054

Kocki T, Wielosz M, Turski WA, Urbanska EM (2006) Enhancement of brain kynurenic acid production by anticonvulsants-novel mechanism of antiepileptic activity? Eur J Pharmacol 541:147-151

Maalouf M, Rho JM, Mattson MP (2009) The neuroprotective properties of calorie restriction, the ketogenic diet, and ketone bodies. Brain Res Rev 59:293-315

Maciejak P, Szyndler J, Turzynska D, Sobolewska A, Taracha E, Skorzewska A, Lehner M, Bidzinski A, Plaznik A (2009) Time course of changes in the concentration of kynurenic acid in the brain of pentylenetetrazol-kindled rats. Brain Res Bull 78:299-305

Mantis JG, Fritz CL, Stephen JM, Heinrichs C, Seyfried TN (2009) Improvement in motor and exploratory behavior in Rett syndrome mice with restricted ketogenic and standard diets. Epilepsy Behav 15:133-141

Moroni F, Russi P, Carla V, Lombardi G (1988) Kynurenic acid is present in the rat brain and its content increases during development and aging processes. Neurosci Lett 94:145-150

Moroni F, Cozzi A, Carpendo R, Cipriani G, Veneroni O, Izzo E (2005) Kynurenine 3-mono-oxygenase inhibitors reduce glutamate concentration in the extracellular spaces of the basal ganglia but not in those of the cortex or hippocampus. Neuropharmacology 48:788-795

Murphy P, Likhodii S, Nylen K, Burnham WM (2004) The antidepressant properties of the ketogenic diet. Biol Psychiatry 56:981-983

Potter MC, Elmer GI, Bergeron R, Albuquerque EX, Guidetti P, Wu HQ, Schwarcz R (2010) Reduction of endogenous kynurenic acid formation enhances extracellular glutamate, hippocampal plasticity, and cognitive behavior. Neuropsychopharmacol 35:1734-1742

Prins ML, Hovda DA (2009) The effects of age and ketogenic diet on local cerebral metabolic rates of glucose after controlled cortical impact injury in rats. J Neurotrauma 26:1083-1093

Ruskin DN, Ross JL, Kawamura M, Ruiz TL, Geiger JD, Masino SA (2011) A ketogenic diet delays weight loss and does not impair working memory or motor function in the R6/2 $1 \mathrm{~J}$ mouse model of Huntington's disease. Physiol Behav 103:501-507

Samala R, Klein J, Borges K (2011) The ketogenic diet changes metabolite levels in hippocampal extracellular fluid. Neurochem Int 58:5-8

Saran T, Hilgier W, Urbanska EM, Turski WA, Albrecht J (2004) Kynurenic acid synthesis in cerebral cortical slices of rats with progressing symptoms of thioacetamide-induced hepatic encephalopathy. J Neurosci Res 75:436-440

Shibata K (1988) Fluorimetric micro-determination of kynurenic acid, an endogenous blocker of neurotoxicity, by high-performance liquid chromatography. J Chromatogr 430:376-380

Stafstrom CE (1999) Animal models of the ketogenic diet: what have we learned, what can we learn? Epilepsy Res 37:241-259

Szyndler J, Maciejak P, Turzynska D, Sobolewska A, Walkowiak J, Plaznik A (2011) The effects of electrical hippocampal kindling of seizures on amino acids and kynurenic acid concentrations in brain structures. J Neural Transm. doi:10.1007/s00702011-0700-z

Thaler S, Choragiewicz TJ, Rejdak R, Fiedorowicz M, Turski WA, Tulidowicz-Bielak M, Zrenner E, Schuettauf F, Zarnowski T (2010) Neuroprotection by acetoacetate and beta-hydroxybutyrate against NMDA-induced RGC damage in rat-possible 
involvement of kynurenic acid. Graefes Arch Clin Exp Ophthalmol 248:1729-1735

Turski WA, Nakamura M, Todd WP, Carpenter BK, Whetsell WO Jr, Schwarcz R (1988) Identification and quantification of kynurenic acid in human brain tissue. Brain Res 454:164-169

Turski WA, Gramsbergen JB, Traitler H, Schwarcz R (1989) Rat brain slices produce and liberate kynurenic acid upon exposure to L-kynurenine. J Neurochem 52:1629-1636

Vamos E, Pardutz A, Klivenyi P, Toldi J, Vecsei L (2009) The role of kynurenines in disorders of the central nervous system: possibilities for neuroprotection. J Neurol Sci 283:21-27

Vecsei L, Beal MF (1990) Intracerebroventricular injection of kynurenic acid, but not kynurenine, induces ataxia and stereotyped behavior in rats. Brain Res Bull 25:623-627
Wejksza K, Rzeski W, Turski WA, Hilgier W, Dybel A, Albrecht J (2006) Ammonia at pathophysiologically relevant concentrations activates kynurenic acid synthesis in cultured astrocytes and neurons. Neurotoxicology 27:619-622

Zadori D, Klivenyi P, Vamos E, Fulop F, Toldi J, Vecsei L (2009) Kynurenines in chronic neurodegenerative disorders: future therapeutic strategies. J Neural Transm 116:1403-1409

Ziegler DR, Gamaro GD, Araujo E, Bassani MG, Perry ML, Dalmaz C, Goncalves CA (2005) Nociception and locomotor activity are increased in ketogenic diet fed rats. Physiol Behav 84:421-427 